\title{
A RELAÇÃO ENTRE SISTEMAS DE COMPENSAÇÃO E MOTIVAÇÃO: UM ESTUDO NA UNIVERSIDADE FEDERAL DO TOCANTINS (UFT)
}

THE RELATIONSHIP BETWEEN SYSTEMS OF COMPENSATION AND MOTIVATION: A STUDY IN THE FEDERAL UNIVERSITY OF TOCANTINS (UFT)

LA RELACIÓN ENTRE LOS SISTEMAS DE COMPENSACIÓN Y MOTIVACIÓN: UN ESTUDIO REALIZADO EN LA UNIVERSIDAD FEDERAL DE TOCANTINS (UFT) Degson Ferreira ${ }^{1}$, Tara Keshar Nanda Baidya ${ }^{2}$, Angilberto Sabino de Freitas ${ }^{3}$

\section{RESUMO}

O presente trabalho tem como objetivo avaliar de que forma um sistema de compensação (remuneração e promoção) exerce influência na motivação dos colaboradores docentes da Universidade Federal do Tocantins (UFT). Para tanto, sistematiza-se um referencial teórico acerca dos aspectos que envolvem a motivação das pessoas, bem como aqueles relacionados às características dos sistemas de recompensas das organizações. A perspectiva de investigação nesta pesquisa é quantitativa de cunho descritivo, adotando-se como procedimento metodológico a realização de uma survey com 290 docentes. Assim, como instrumento de coleta de dados, utilizou-se da aplicação de questionários estruturados. A análise e interpretação dos dados, realizadas com o uso de estatísticas descritivas como a análise múltipla de regressão, permitem destacar que a transparência das oportunidades de progressão e a equidade do sistema de remuneração são variáveis que exercem um efeito positivo estatisticamente significativo tanto na motivação extrínseca quanto na motivação intrínseca dos docentes da UFT. Entre as variáveis independentes que não afetam de forma significativa estatisticamente nenhuma das variáveis dependentes estão transparência do sistema de remuneração e a controlabilidade das oportunidades de progressão.

PALAVRAS-CHAVE: Sistemas de Recompensas. Características dos Sistemas de Recompensas. Motivação Extrínseca. Motivação Intrínseca. Universidade Federal do Tocantins.

\begin{abstract}
This paper aims to assess how a system of compensation (pay and promotion) influences the motivation of teaching staff of the Federal University of Tocantins (UFT). For both, a theoretical reference is organized about the issues involving people's motivation, as well as those related to the characteristics of the organizations's rewards systems. The prospect of research in this paper is quantitative, descriptive stamp. It was adopted as a methodological procedure to carry out a survey of 290 teachers. Thus, as data collection instrument was used application of structured questionnaires. The analysis and interpretation of data, performed using descriptive statistics such as multiple regression analysis, allow to point out that the transparency of progression opportunities and the fairness of the remuneration system are variables that exert a statistically significant positive effect on both the extrinsic motivation as the intrinsic motivation of the UFT's teachers. Among the independent variables that do not statistically significantly affect any of the dependent variables are
\end{abstract}

\footnotetext{
${ }^{1}$ Doutorando em Administração - Universidade do Grande Rio (UNIGRANRIO) - Rio de Janeiro, RJ. Brasil. Professor efetivo Adjunto I - Curso de Logística da Universidade Federal do Tocantins (UFT) - Tocantins, MT, Brasil. E-mail: degsonfer@gmail.com

${ }^{2}$ Doutor em Administração de Empresas - University of California, Berkeley, EUA; Professor do Doutorado em Administração da Universidade do Grande Rio (UNIGRANRIO) - Rio de Janeiro, RJ. Brasil. E-mail: tarabaidya@yahoo.com.br

${ }^{3}$ Doutor em Administração de Empresas - Pontifícia Universidade Católica do Rio de Janeiro (PUC - Rio) - Rio de Janeiro, RJ. Brasil; Professor do Doutorado em Administração da Universidade do Grande Rio (UNIGRANRIO) - Rio de Janeiro, RJ. Brasil. E-mail: angilberto.freitas@gmail.com
}

Submetido em: 06/09/2016 - Aceito em: 02/12/2017

(C) ETD- Educação Temática Digital Campinas, SP $\quad$ v.20 $\quad$ n.1 $\quad$ p. 27-48 jan./mar. 2018 
transparency of the remuneration system and the controllability of progression opportunities.

KEYWORDS: Rewards Systems. Characteristics Rewards Systems. Extrinsic Motivation. Intrinsic Motivation. Federal University of Tocantins.

\section{RESUMEN}

Este artículo tiene como objetivo evaluar cómo un sistema de compensación (paga y promoción) influye en la motivación de los empleados de enseñanza de la Universidad Federal de Tocantins (UFT). Por lo tanto, un marco teórico se organiza en los aspectos relativos a la motivación de las personas, así como los relacionados con las características de los sistemas de recompensa de las organizaciones. La perspectiva de la investigación en este artículo es cuantitativa de la naturaleza descriptiva. Fue adoptado como un procedimiento metodológico para llevar a cabo una encuesta de 290 maestros. De este modo, como instrumento de recolección de datos se utilizó la aplicación de cuestionarios estructurados. El análisis e interpretación de datos, realiza con el uso de la estadística descriptiva, como el análisis de regresión múltiple, permiten señalar que la transparencia de las oportunidades de progresión y la equidad del sistema de remuneración son variables que juegan un efecto positivo estadísticamente significativo tanto en la motivación extrínseca como la motivación intrínseca de los maestros UFT. Entre las variables independientes que no afectan de manera significativa estadísticamente ninguna de las variables dependientes son la transparencia del sistema de remuneración y la controlabilidad de las oportunidades de progresión.

PALABRAS CLAVE: Sistemas de Recompensa. Características de los Sistemas de Recompensa. Motivación Extrínseca. Motivación Intrínseca. Universidad Federal de Tocantins.

\section{INTRODUÇÃO}

Dentre todas as questões administrativas referentes à motivação, possivelmente uma que recebe atenção especial é o caso dos sistemas de compensação (MARRAS, 2011). Isso porque os sistemas de recompensas (remuneração, salários, benefícios, promoções, etc.) podem constituir-se em um dos principais fatores de motivação dos indivíduos nas organizações, conforme destacam autores como Jessup (1977), Bergamini (1992, 2008), Harris (1997), Davis e Newstrom (1998), Wood Jr. e Picarelli Filho (1999), Gomes e Amorim (2008), França (2009), Vieira e Souza (2010), Câmara (2011), Alves e Pinto (2011), Cardoso, Cardoso e Santos (2013), Duarte e Melo (2013), Vieira e Oliveira (2013).

Os trabalhos desenvolvidos por esses autores podem ser caracterizados, em sua maioria, como sendo pesquisas de caráter exploratório e descritivo e apenas ressaltam, de forma argumentativa e sem o uso de técnicas para estabelecer inferências, a influência de diversos fatores tanto internos quanto externos na motivação dos colaboradores.

Dado esse contexto de caracterização dos trabalhos desenvolvidos, as pesquisas nacionais, de maneira geral, em consonância com as estruturas conceituais e paradigmáticas vigentes ao longo dos anos no país, focalizaram-se em investigações dos fatores intervenientes da motivação, utilizando como base, prioritariamente, instrumentos e metodologias de pesquisas de natureza qualitativa. 
Então, pode-se destacar que há poucos estudos empíricos, dentre eles o de Van Herpen (2007), que analisam e testam estatisticamente os efeitos dos sistemas de compensação (salários, benefícios, promoções, etc.) sobre a motivação no trabalho. No sentido de providenciar informações para o preenchimento dessa lacuna, mas não de exaurir a possibilidade de tratamento sobre o tema, é que se insere a proposta deste artigo: avaliar em que medida um sistema de compensação (remuneração e promoção) exerce influência na motivação dos colaboradores docentes da Universidade Federal do Tocantins (UFT).

Nesse sentido, este trabalho se justifica pela tentativa de preencher essa fenda teórica, concentrando-se em testar, medir e avaliar estatisticamente os efeitos dos sistemas de compensação (remuneração e promoções) sobre a motivação docente; concomitantemente, esta pesquisa também assume sua relevância e originalidade por se concentrar em analisar a relação entre estes fatores e a sua influência na motivação docente por meio de uma abordagem ainda não tratada na literatura brasileira, abordagem quantitativa desta relação que caracteriza o problema de pesquisa.

\section{MOTIVAÇÃO EXTRÍNSECA E INTRÍNSECA}

A motivação é um dos fenômenos mais complexos no estudo do comportamento humano, e é difícil defini-la de modo preciso, uma vez que tem sido utilizada com diferentes sentidos. Porém, apesar da pouca diferenciação significativa na estruturação da redação, há um consenso na definição da motivação como sendo o conjunto de processos implicados no impulso, na direção, na intensidade e na persistência do comportamento ou conduta (MITCHELL, 1982; PETRI, 1996; DÖRNYEI, 2000; TAMAYO e PASCHOAL, 2003).

Vários autores também compartilham a ideia de que, no sentido lato, o termo motivação significa a ação ou o efeito de motivar, de dar motivos, de estimular o surgimento de interesses por algo ou para a vida das pessoas, de impulsionar a pessoa a apresentar um determinado comportamento (KAST e ROSENZWEIG, 1970; STONER e FREEMAN, 1985; FERREIRA, 2001; CAVALCANTI et al., 2005; SALGADO, 2005; CASTRO, 2008).

Assim, pode-se destacar que existem dois tipos de motivação, inicialmente identificados por Deci (1972) e posteriormente estudados por outros autores, tais como Frey e Jegen (2001) e Bénabou e Tirole (2003): a extrínseca e a intrínseca. A motivação extrínseca ocorre quando uma atividade é efetuada com outro objetivo que não o inerente à própria atividade; ela é afetada por valores externos à atividade, estando presente quando a realização de uma atividade por um indivíduo objetiva a obtenção de uma recompensa

(C) ETD- Educação Temática Digital $\quad$ Campinas, SP $\quad$ v.20 $\quad$ n.1 $\quad$ p. 27-48 jan./mar. 2018


externa à atividade ou quando ele realiza essa atividade para evitar o fracasso (DECl e RYAN, 2000).

A motivação intrínseca, por sua vez, é caracterizada pelo envolvimento e compromisso do indivíduo com a atividade por si só, devido ao prazer e gosto que ela produz, representando a atividade, nessas circunstâncias, um fim em si mesmo.Os indivíduos quando estão motivados intrinsecamente são movidos para a ação pelo interesse, diversão ou satisfação que the é inerente, em detrimento das recompensas ou pressões externas para realizar a mesma atividade (DECl e RYAN, 2000; BÉNABOU e TIROLE, 2003).

\section{CARACTERÍSTICAS DOS SISTEMAS DE COMPENSAÇÃO}

O sistema de recompensas inclui o pacote total de benefícios que a organização coloca à disposição de seus colaboradores e os procedimentos pelos quais esses benefícios são distribuídos. Inclui não apenas salários, férias, oportunidades de promoções, mas também recompensas como garantia de segurança no cargo, transferências para posições mais desafiantes e as várias formas de reconhecimento por serviços prestados (MILES, 1975).

Chiang e Birtch (2006), ao identificarem diversos tipos de recompensas, fizeram uma conjugação entre recompensas financeiras e não financeiras com recompensas extrínsecas e intrínsecas. Para eles, as recompensas financeiras são consideradas extrínsecas, haja vista referirem-se aos reforços externos de natureza monetária e incluírem benefícios diretos os pagamentos que os funcionários recebem como bônus, prêmios e a retribuição em dinheiro pela execução de seus serviços - e indiretos - decorrentes dos acordos coletivos de trabalho e dos programas de benefícios da organização como férias, gratificações, participação nos resultados, auxílio alimentação, transporte, seguro de saúde, etc.

As recompensas não financeiras não beneficiam os colaboradores em termos monetários e podem ser de duas naturezas: intrínseca ou extrínseca. As recompensas não financeiras extrínsecas são reforços externos que a organização utiliza para estimular o bom desempenho do profissional no trabalho que são mediadas fora da pessoa como, por exemplo, as relações com os colegas de trabalho, as oportunidades de promoções, status, participação na tomada de decisão, estilo de gestão, formação e desenvolvimento profissional, equilíbrio entre vida pessoal e profissional, disponibilidade de recursos suficientes para a execução do trabalho, reconhecimento e ambiente de trabalho favorável (CHIANG e BIRTCH, 2006). 
As recompensas não financeiras intrínsecas são aquelas que envolvem experiências individuais como sentimento de competência ao realizar um bom trabalho e estão relacionadas com recompensas psicológicas. São mediadas dentro da pessoa e podem estar na natureza desafiadora do trabalho, na responsabilidade, na possibilidade de exercer as suas competências, na autonomia, no orgulho de suas habilidades, na realização e na satisfação no trabalho (CHIANG e BIRTCH, 2006; MEDEIROS, ALVES e RIBEIRO, 2012).

Levando em consideração essa classificação e a conjugação realizada por Chiang e Birtch (2006), propõe-se a Figura 1 para representar uma visão mais diversificada e sistêmica da natureza financeira e não financeira, extrínseca e intrínseca das recompensas.

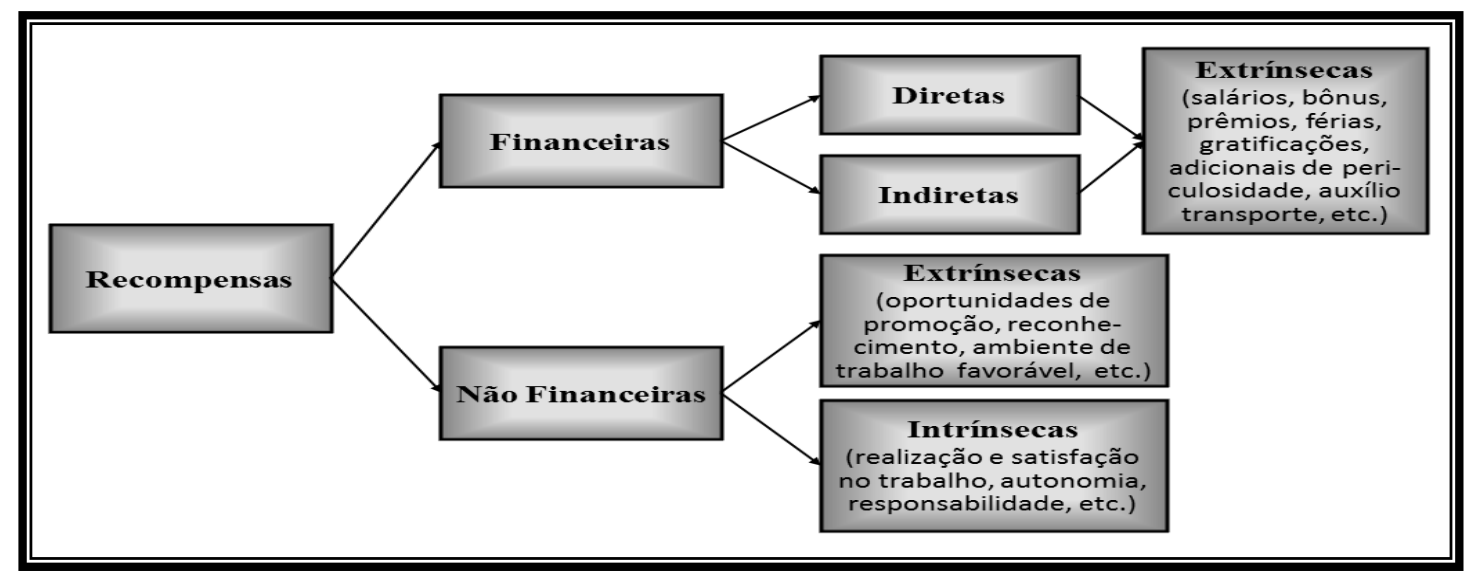

Figura 1 - Tipologia de Recompensas Ampliada

Fonte: Autores, baseados em Dutra (2002), Chiang e Birtch (2006) e Câmara (2011).

De acordo com essas considerações realizadas e, em paralelo com Câmara (2011), pode-se destacar que para que um sistema de compensação seja eficaz e consiga alcançar os objetivos para o qual foi criado, é fundamental que ele possua algumas características como, por exemplo, que esteja alinhado com os objetivos estratégicos da organização, e que seja bem aceito pelos seus destinatários e percebido por eles como sendo justo e objetivo, funcionando de uma forma equitativa e avaliando a contribuição de cada um, de acordo com critérios claros e incontroversos.

Para Thierry (1987) apresentado em Van Herpen (2007), a eficácia de um sistema de compensação (aqui assumido como a combinação de recompensas monetárias e possibilidades de promoção) depende de três características percebidas, a saber: (1) transparência, (2) justiça e (3) controlabilidade.

Para Van Herpen (2007), a transparência percebida de um sistema de compensação depende de duas características: comunicação adequada e complexidade. Para o autor, um sistema de comunicação transparente, além de informar os objetivos que a organização

(C) ETD- Educação Temática Digital Campinas, SP $\quad$ v.20 $\quad$ n.1 $\quad$ p. 27-48 jan./mar. 2018


pretende alcançar, também informa aos seus colaboradores as regras do sistema de compensação por meio de uma comunicação clara dessas regras, permitindo-lhes uma melhor compreensão das metodologias, indicadores e metas a serem utilizadas.

A teoria da equidade enfatiza a necessidade do indivíduo receber uma quantidade razoável de compensação em relação a outros indivíduos. Isso porque eles comparam a proporção entre seus inputs (esforços) e os resultados (recompensas) com a relação inputresultado de outros indivíduos. Se um indivíduo enxergar essa relação como desigual, um estado de tensão é provocado, o qual, por sua vez, levará a uma tentativa de restaurar a igualdade.Janssen (2001) mostrou empiricamente que os gestores que percebem justiça na relação esforço-recompensa desempenham melhor do que aqueles que percebem que estão injustamente recompensados.

A terceira característica do sistema de compensação é a relação percebida entre esforço e compensação, também denominada de controlabilidade do sistema. Van Herpen (2007) destaca que Baker (2002) define controlabilidade como a extensão em que o indivíduo é capaz de controlar ou influenciar o resultado de suas ações.

\section{PROCEDIMENTOS METODOLÓGICOS}

O presente trabalho classifica-se como pesquisa descritiva (CERVO e BERVIAN, 2002; GIL, 2002; KÖCHE, 2002; LEITE, 2004; MALHOTRA, 2012), pois visa transcrever, descrever, interpretar e explicar o resultado do estudo obtido na avaliação da forma como a concepção de um sistema de compensação (remuneração e promoção) influencia a motivação dos colaboradores docentes da Universidade Federal do Tocantins - UFT.

Tendo em vista a natureza do problema investigado, a abordagem de pesquisa utilizada foi a quantitativa. Este tipo de abordagem de pesquisa, segundo Richardson et al. (1999), caracteriza-se pelo emprego da quantificação tanto na coleta das informações quanto no seu tratamento por meio de ferramentas estatísticas. Para os autores, sua finalidade básica é garantir a precisão dos resultados, evitar distorções de análise e de interpretação, atribuindo aos resultados obtidos uma margem maior de confiança.

Para Bryman (2004), as pesquisas quantitativas são geralmente conceituadas por seus adotantes como tendo uma estrutura lógica na qual as teorias determinam o problema para o qual os pesquisadores as endereçam na forma de hipóteses derivadas de teorias gerais. 


\subsection{Declaração do Problema, Questão de Investigação e Hipóteses}

Como mencionado na parte introdutória deste estudo, tem-se como problema de investigação verificar

\footnotetext{
de que forma a concepção de um sistema de compensação (remuneração e promoção)influencia a motivação dos colaboradores docentes da Universidade Federal do Tocantins - UFT?
}

A opção pela investigação desses fatores que influenciam a motivação decorre da crescente importância que eles vêm conquistando no cenário nacional e internacional e da carência de estudos específicos para medir e testar estatisticamente essa relação entre sistemas de compensação e sua influência na motivação dos indivíduos.

Entretanto, parte da literatura vem apontando que o sistema de compensação é utilizado pelas organizações como uma forma de atrair e reter seus talentos, reduzir a rotatividade de pessoal, diminuir o absenteísmo, aumentar a produtividade e motivar seus colaboradores, conseguindo deles uma contrapartida correspondente às suas competências. Autores como Bergamini (1992), Harris (1997), Marras (2000), Dutra (2002), Bohlander e Snell (2009) e Câmara (2011) compartilham dessa ideia.

Para que um sistema de compensação seja eficaz e consiga alcançar os objetivos para o qual foi criado, é fundamental que ele possua algumas características. Thierry (1987) apresentado em Van Herpen (2007) indica que a transparência, a justiça e a controlabilidade são características que levam à eficácia do sistema de compensação de uma organização.Esta última visão também é compartilhada por autores como Bergamini (1992), Harris (1997) e Bohlander e Snell (2009).

Autores como Albuquerque (1999), Dutra (2002), Hellriegel e Slocum Jr. (2004) e Bohlander e Snell (2009) destacam que fatores internos e externos importantes relacionados com o trabalho, que levam à motivação e à satisfação no emprego, incluem desafios no cargo, supervisão competente, remuneração equitativa e justa e carreiras recompensadoras. Assim, espera-se que as características do sistema de remuneração (transparência, justiça e controlabilidade) exerçam um impacto sobre o nível de motivação dos colaboradores.

A literatura ainda destaca que a oportunidade de ganhar uma promoção além de permitir à organização utilizar as habilidades e as capacidades de seu pessoal de maneira mais efetiva, também serve como incentivo para o bom desempenho e o aumento da satisfação. Van Herpen (2007) ressalta que além de um aumento salarial, uma promoção aumenta o status do funcionário e pode trazer novos desafios que fortalecem a sua motivação intrínseca. Por isso, a opção por testar a hipótese da relação entre as

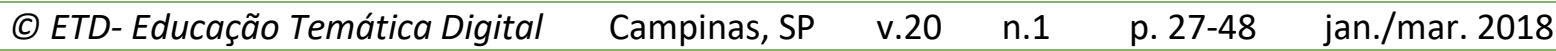


características das oportunidades de promoção (progressão) e a motivação extrínseca e intrínseca dos colaboradores da UFT.

Estas dimensões emergem-se da literatura revisada como elementos que devem exercer influência determinante na configuração da motivação das pessoas. Por este motivo, dão sustentação à questão de investigação, estruturada para a pesquisa empírica e que é apresentada, juntamente com suas hipóteses, a seguir.

Questão de Investigação:

Qual é o efeito do sistema de compensação (remuneração e oportunidades de progressão) sobre a motivação dos colaboradores docentes da UFT?

\section{Hipóteses:}

$\mathrm{H}_{1 \mathrm{a}}$ : Quanto maior a percepção da transparência do sistema de remuneração, maior a motivação extrínseca dos colaboradores docentes da UFT.

$\mathrm{H}_{1 \mathrm{~b}}$ : Quanto maior a percepção da equidade do sistema de remuneração, maior a motivação extrínseca dos colaboradores docentes da UFT.

$\mathrm{H}_{1 c}$ : Quanto maior a percepção da controlabilidade do sistema de remuneração, maior a motivação extrínseca dos colaboradores docentes da UFT.

$\mathrm{H}_{2 a}$ : Quanto maior a percepção da transparência das oportunidades de progressão, maior a motivação extrínseca dos colaboradores docentes da UFT.

$\mathrm{H}_{2 \mathrm{~b}}$ : Quanto maior a percepção da equidade das oportunidades de progressão, maior a motivação extrínseca dos colaboradores docentes da UFT.

$\mathrm{H}_{2 c}$ : Quanto maior a percepção da controlabilidade das oportunidades de progressão, maior a motivação extrínseca dos colaboradores docentes da UFT.

$\mathrm{H}_{3 a}$ : Quanto maior a percepção da transparência do sistema de remuneração, maior a motivação intrínseca dos colaboradores docentes da UFT.

$\mathrm{H}_{3 \mathrm{~b}}$ : Quanto maior a percepção da equidade do sistema de remuneração, maior a motivação intrínseca dos colaboradores docentes da UFT.

$\mathrm{H}_{3 c}$ : Quanto maior a percepção da controlabilidade do sistema de remuneração, maior a motivação intrínseca dos colaboradores docentes da UFT.

$\mathrm{H}_{4 a}$ : Quanto maior a percepção da transparência das oportunidades de progressão, maior a motivação intrínseca dos colaboradores docentes da UFT. 
$\mathrm{H}_{4 \mathrm{~b}}$ : Quanto maior a percepção da equidade das oportunidades de progressão, maior a motivação intrínseca dos colaboradores docentes da UFT.

$\mathrm{H}_{4 c}$ : Quanto maior a percepção da controlabilidade das oportunidades de progressão, maior a motivação intrínseca dos colaboradores docentes da UFT.

\subsection{Universo e Amostra da Pesquisa}

A delimitação da amostra se deu através do processo denominado de amostragem não-probabilística intencional (KIDDER et al., 1987). Assim, a pesquisa foi realizada junto aos professores lotados nos campi da UFT das cidades de Araguaína e de Palmas por sua representatividade e significância, pois aproximadamente $67,6 \%$ do quadro docente desta instituição concentram-se nesses dois campi.

Por meio de informações obtidas junto ao Setor de Desenvolvimento Humano (SDH, 2014) da UFT, pôde-se verificar que o universo da pesquisa constitui-se de 632 professores. Sendo assim, neste espaço delimitado para estudo, retirando os professores em que foi aplicado o pré-teste do instrumento de coleta de dados, bem como aqueles que não foram localizados para a entrega ou envio do questionário e aqueles que não aceitaram participar da pesquisa, somente 290 participaram da pesquisa e enquadraram nos critérios e qualificações pré-determinados para a mesma, constituindo, desta forma, a amostra final da pesquisa.

Em relação à distribuição geográfica dos docentes da UFT que foram pesquisados, a maioria deles, cerca de 54,83\% (159), está localizada no campus da cidade de Palmas. Os outros 45,17\% (131) docentes estão distribuídos no campus da cidade de Araguaína.

\subsection{Coleta, Análise e Tratamento dos Dados}

A coleta de dados caracterizou pelo trabalho de busca de informações na UFT, utilizando-se como instrumento de pesquisa, para alcançar os objetivos propostos, a aplicação de questionários(LAKATOS e MARCONI, 2005). De forma analítica, os questionários continham perguntas fechadas ou dicotômicas do tipo "sim" ou "não"; e perguntas fechadas que combinam com respostas abertas. Mediante o questionário, as questões foram respondidas por escrito pelos pesquisados, sem a intermediação ou a presença do pesquisador.

Todas as perguntas relacionadas à percepção dos colaboradores sobre o sistema de compensação da UFT, bem como as que dizem respeito à sua motivação extrínseca e intrínseca foram formuladas como assertivas. Os colaboradores foram convidados a reagir a essas afirmações, em uma escala tipo Likert, fornecendo respostas que poderiam variar de 1 a 5 , sendo " 1 ", equivalente a discordo e " 5 ", correspondendo a concordo.

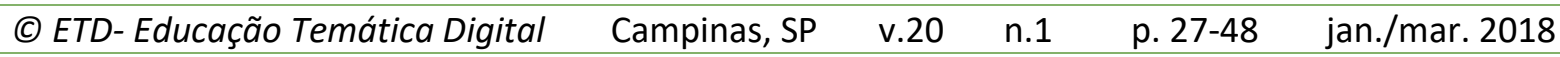


Após a coleta dos dados, eles foram tabulados e classificados de forma sistemática por meio de estatísticas descritivas como, por exemplo, com a realização de análise de regressão linear múltipla. $\mathrm{Na}$ análise e interpretação dos dados utilizou-se de instrumentos de apoio como os softwares Microsoft Excel ${ }^{\circledR}$ e IBM $^{\circledR}$ SPSS $^{\circledR}$ (Statistical Package for the Social Sciences), versão 20.0, executando em ambiente Windows ${ }^{\circledR}$, para dar um significado mais amplo às respostas, vinculando-as a outros conhecimentos.

\section{RESULTADOS E DISCUSSÕES}

Para identificar o efeito que as características do sistema de compensação da UFT (transparência do sistema de remuneração, transparência das oportunidades de progressão, equidade do sistema de remuneração, equidade das oportunidades de progressão, controlabilidade do sistema de remuneração e controlabilidade das oportunidades de progressão - variáveis independentes) exercem sobre a motivação extrínseca e intrínseca (variável dependente) de seu pessoal docente, a técnica de regressão múltipla foi utilizada. Como as características individuais podem ter um impacto sobre a relação entre as variáveis, variáveis de controle foram usadas em cada uma das análises realizadas. Os resultados da relação entre as características do sistema de compensação e a motivação extrínseca dos docentes da UFT podem ser visualizados na Tabela 1.

Na Tabela 1, pode-se notar que a análise de regressão múltipla revelou que somente a transparência das oportunidades de progressão (Transparência_OP), a equidade do sistema de remuneração (Equidade_SR), a equidade das oportunidades de progressão (Equidade_OP) e a controlabilidade do sistema de remuneração (Controlabilidade_SR) são as variáveis independentes que contribuem substancialmente para o modelo prever um resultado. Entre as variáveis de controle, somente o tempo na instituição (dado em anos) e a existência de metas claras a cumprir são variáveis significativas para o modelo.

O coeficiente de determinação ou de regressão do modelo $\left(R^{2}\right)$ resultante é 0,673 , o qual é significativo ao nível de $0,1 \%$. Isso significa que, na amostra, $67,3 \%$ da variação nos níveis da motivação extrínseca dos docentes da UFT pode ser atribuída às variáveis independentes e de controle. Uma das condições que precisa ser satisfeita na análise de regressão é a de que os erros devem ser não-correlacionados (ou independentes). Essa suposição foi verificada com o teste proposto por Durbin e Watson (1951), denominado de Durbin-Watson, que neste caso por ser igual a 2,089 (bem perto de 2) indica que os erros não devem estar correlacionados entre si ou, em outros termos, são independentes. 
TABELA 1 - Influência das Características do Sistema de Compensação na Motivação Extrínseca

\begin{tabular}{l|r|r|c}
\hline & $\boldsymbol{B}$ & $\mathbf{S}_{\mathrm{e}} \mathbf{B}$ & $\mathbf{B}$ \\
\hline Constante & 0,287 & 0,146 & $-0,003$ \\
Transparência_SR & $-0,003$ & 0,046 & $0,133^{* *}$ \\
Transparência_OP & 0,108 & 0,031 & $0,538^{* * *}$ \\
Equidade_SR & 0,463 & 0,037 & $0,147^{* *}$ \\
Equidade_OP & 0,130 & 0,038 & $0,223^{* * *}$ \\
Controlabilidade_SR & 0,201 & 0,035 & 0,079 \\
Controlabilidade_OP & 0,069 & 0,042 & $-0,114^{* *}$ \\
Tempo na Instituição (em anos) & $-0,029$ & 0,009 & $0,093^{* *}$ \\
Existência de Metas & 0,219 & 0,081 & \\
\hline Sig. & $\mathbf{p < 0 , 0 0 1}$ & \\
$\mathbf{R}^{\mathbf{2}}$ & $\mathbf{0 , 6 7 3}$ & \\
$\mathbf{R}^{\mathbf{2}}$ Ajustado & $\mathbf{0 , 6 6 6}$ & \\
\hline
\end{tabular}

Nota: $\boldsymbol{B}=$ coeficiente de regressão não padronizado; $\mathbf{S}_{\mathrm{e}} \boldsymbol{B}=$ erro padrão da estimativa (mede o desvio médio entre os valores reais de $\mathrm{Y}$ e os valores estimados $\boldsymbol{Y}^{\prime}$ ); $\boldsymbol{B}=$ coeficiente de regressão padronizado.

$* * * p<0,001 \quad * * p<0,01$

Fonte: Dados primários (2016)

O coeficiente não padronizado da transparência do sistema de remuneração (Transparência_SR) mostrado na Tabela 1 dos coeficientes é $-0,003$. Esse valor indica que quando a transparência desse sistema aumenta uma unidade, a motivação extrínseca diminui 0,003, apresentando uma relação inversa com esta variável dependente. Entretanto, com o valor $t$ de-0,058e com uma probabilidade associada de 0,954, [t (272) = $0,058, p>0,05]$, pode-se afirmar que a mudança nos níveis de motivação é a mesma se o escore da transparência do sistema de remuneração aumentar uma unidade. Portanto, os níveis de motivação extrínseca dos docentes da UFT pesquisados não diminuem e nem aumentam em decorrência do aumento na transparência do seu sistema de remuneração, o que, por conseguinte, leva a refutação da hipótese 1 .

No caso da influência da transparência das oportunidades de progressão (Transparência_OP) sobre a motivação extrínseca, os resultados evidenciaram que os níveis de motivação extrínseca dos docentes da UFT pesquisados aumentam 0,108 graus em decorrência do aumento de uma unidade na transparência dessas oportunidades, pois a estatística $t$ mostrou-se significativa $[t(283)=3,490, p<0,01]$, corroborando a hipótese 2 a.

No que se refere ao efeito da equidade do sistema de remuneração (Equidade_SR) sobre a motivação extrínseca, os resultados demonstraram que os níveis de motivação extrínseca dos docentes da UFT pesquisados aumentam 0,463 graus em decorrência do aumento, em média, de uma unidade na equidade desse sistema, haja vista que o valor da estatística $t$ apresenta-se de forma muito significativa $[t(283)=12,672, p<0,001]$, levando a confirmação da hipótese $1 \mathrm{~b}$. 
Em relação ao efeito da equidade das oportunidades de progressão (Equidade_OP) sobre a motivação extrínseca, pode-se afirmar com base nos resultados apresentados que os níveis de motivação extrínseca dos docentes da UFT pesquisados aumentam 0,130 graus em decorrência do aumento de uma unidade na equidade dessas oportunidades. $O$ valor da estatística $t$ confirma esses resultados ao mostrar-se de forma bastante significativa [ $t$ (283) $=3,439, p<0,01]$, suportando, desse modo, a hipótese $2 b$.

No que diz respeito à influência da controlabilidade do sistema de remuneração sobre a motivação extrínseca, os resultados permitem dizer que os níveis de motivação extrínseca dos docentes da UFT aumentam 0,201 graus em decorrência do aumento de uma unidade na controlabilidade desse sistema. Como o valor do teste $t$ mostrou ser muito significativo [ $t(283)=5,741, p<0,001]$, essa mudança nos níveis de motivação extrínseca dos docentes da UFT pode ser confirmada, o que, por conseguinte, corrobora a hipótese 1c.

No tocante à relação entre a controlabilidade das oportunidades de progressão (Controlabilidade_OP) e a motivação, os resultados encontrados evidenciaram que os níveis de motivação extrínseca dos docentes da UFT realmente não aumentam em decorrência do aumento de uma unidade na controlabilidade dessas oportunidades, pois o valor da estatística $t$ não é significativo [ $t(272)=1,633, p>0,05]$, levando a rejeição da hipótese $2 c$.

Entre as variáveis de controle, conforme pode ser visualizado na Tabela 1, somente o tempo de atuação na instituição (dado em anos) e a existência de metas mostraram ser variáveis significativas para o modelo. No que concerne ao efeito do tempo de atuação dos docentes na instituição sobre a motivação, os resultados permitem enfatizar que os níveis de motivação extrínseca dos docentes da UFT diminuem 0,029 graus em decorrência do aumento, em média, de uma unidade (no caso um ano) no tempo de suas atuações na instituição. $O$ valor da estatística $t$ confirma esses resultados ao revelar-se de forma bastante significativa $[t(283)=-3,233, p<0,01]$.

O coeficiente não padronizado da existência de metas mostrado na tabela dos coeficientes é 0,219 . Como essa variável é uma dummy, onde 0 significa ausência de metas explícitas a cumprir e 1 significa a existência de metas claras a cumprir, esse valor do coeficiente indica que sea existência de metas aumentar em uma unidade, no caso passar de 0 para 1 (passar a ter metas claras a cumprir), a motivação extrínseca aumentará 0,219, apresentando uma relação positiva com esta variável de controle. Com o valor $t$ de2,718 e com uma probabilidade significativa associada de $0,007,[t(283)=2,718, p<0,01]$, pode-se afirmar que a mudança nos níveis de motivação realmente é diferente se o escore da existência de metas aumentar uma unidade. Portanto, pode-se dizer que os níveis de motivação extrínseca dos docentes da UFT aumentam como resultado da existência de

(C) ETD- Educação Temática Digital $\quad$ Campinas, SP $\quad$ v.20 $\quad$ n.1 $\quad$ p. 27-48 jan./mar. 2018


metas explícitas a cumprir. Essa interpretação é verdadeira somente se os efeitos dos outros previsores significativos forem mantidos constantes.

$\mathrm{Na}$ Tabela 1 , os coeficientes padronizados ou beta $(\beta)$ da transparência das oportunidades de progressão (Transparência_OP) e da equidade das oportunidades de progressão (Equidade_OP) mostrados na tabela dos coeficientes estão bem próximos, indicando que as duas variáveis apresentam um grau de importância comparável para o modelo. Além disso, a magnitude da estatística $t$ também confirma essa proximidade. $\mathrm{Na}$ comparação entre as variáveis independentes para identificar a mais importante para o modelo, a variável que mais explica a motivação extrínseca dos professores da UFT é a equidade do sistema de remuneração, com o coeficiente padronizado de 0,538 , além do que a magnitude da estatística $t$ de 12,672 confirma essa importância para a variável dependente.

Em relação à multicolinearidade nos dados, observando os valores apresentados pelo Fator de Inflação da Variância (FIV) e pela estatística Tolerância e em paralelo com as observações de Meyers (1990), Menard (2002) e Field (2009), pode-se destacar que todos os valores de FIV estão abaixo de 10 e todas as Tolerâncias estão acima de 0,20. Portanto, pode-se concluir com segurança que não existe multicolinearidade (variáveis altamente correlacionadas) dentro do conjunto de dados observados.

Portanto, de forma resumida, pode-se enfatizar que as variáveis transparência das oportunidades de progressão, equidade do sistema de remuneração, equidade das oportunidades de progressão, controlabilidade do sistema de remuneração, tempo de atuação na instituição e existência de metas claras a cumprir exercem um efeito significativo na motivação extrínseca dos docentes da UFT pesquisados. Porém, as variáveis transparência do sistema de remuneração e controlabilidade das oportunidades de progressão não afetam de forma significativa a motivação extrínseca desses professores.

A regressão múltipla também foi utilizada para verificar como as características do sistema de compensação da UFT afetam a motivação intrínseca (variável dependente) de seu pessoal docente. Variáveis de controle também foram usadas para tentar controlar o impacto das características individuais. Os resultados da relação entre as características do sistema de compensação e a motivação intrínseca dos docentes da UFT podem ser vistos na Tabela 2.

Pela visualização da Tabela 2, percebe-se que a análise de regressão mostrou que somente a transparência das oportunidades de progressão (Transparência_OP) e a equidade do sistema de remuneração (Equidade_SR) são as variáveis independentes que contribuem 
substancialmente para o modelo prever um resultado. Entre as variáveis de controle, somente a idade e o estado civil mostraram ser variáveis significativas no modelo.

O coeficiente de determinação $\left(R^{2}\right)$ resultante é 0,223 , significativo ao nível de $0,1 \%$. Isso significa que, na amostra, $22,3 \%$ da variação nos níveis da motivação intrínseca dos docentes da UFT pode ser atribuída à variação nas variáveis independentes e de controle. A suposição de que os erros não estão correlacionados ou são independentes foi checada com a estatística de Durbin-Watson, a qual é igual a 2,091 (bem perto de 2 e maior do que 1,5), indicando que esta suposição certamente deve estar satisfeita.

TABELA 2 - Influência das Características do Sistema de Compensação na Motivação Intrínseca

\begin{tabular}{|c|c|c|c|}
\hline & $B$ & $\mathrm{~S}_{\mathrm{e}} B$ & B \\
\hline Constante & 3,162 & 0,173 & \\
\hline Transparência_SR & $-0,034$ & 0,040 & $-0,072$ \\
\hline Transparência_OP & 0,110 & 0,025 & $0,237 * * *$ \\
\hline Equidade_SR & 0,147 & 0,027 & $0,300 * * *$ \\
\hline Equidade_OP & 0,018 & 0,034 & 0,036 \\
\hline Controlabilidade_SR & 0,041 & 0,036 & 0,079 \\
\hline Controlabilidade_OP & 0,033 & 0,037 & 0,065 \\
\hline Idade & 0,009 & 0,003 & $0,132 *$ \\
\hline Estado Civil & $-0,072$ & 0,026 & $-0,145^{* *}$ \\
\hline \multicolumn{4}{|c|}{$p<0,001$} \\
\hline $\mathrm{R}^{2}$ & \multicolumn{3}{|l|}{0,223} \\
\hline$R^{2}$ Ajustado & \multicolumn{3}{|l|}{0,212} \\
\hline
\end{tabular}

Nota: $\boldsymbol{B}$ = coeficiente de regressão não padronizado; $\mathbf{S}_{\mathrm{e}} \boldsymbol{B}=$ erro padrão da estimativa (mede o desvio médio

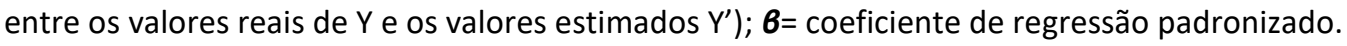

$* * * p<0,001 \quad * * p<0,01 \quad * p<0,05$

Fonte: Dados primários (2016)

O coeficiente não padronizado da transparência do sistema de remuneração (Transparência_SR) mostrado na Tabela 2 dos coeficientes é -0,034. Esse valor indica que quando a transparência desse sistema aumenta em uma unidade, a motivação intrínseca diminui 0,034 níveis, apresentando uma relação inversa com esta variável dependente. Entretanto, com o valor $t$ de-0,847e com uma probabilidade associada de 0,398 [ $t(272)=-$ $0,847, p>0,05]$, pode-se afirmar que a mudança nos níveis de motivação intrínseca é a mesma se o escore da transparência do sistema de remuneração aumentar em uma unidade. Portanto, os níveis de motivação intrínseca dos docentes da UFT pesquisados não diminuem e nem aumentam em decorrência do aumento na transparência do sistema de remuneração, o que, por conseguinte, leva a rejeição da hipótese $3 a$.

No caso do efeito da transparência das oportunidades de progressão sobre a motivação intrínseca, os resultados evidenciaram que os níveis de motivação intrínseca dos docentes da UFT aumentam 0,110 graus em decorrência do aumento de uma unidade na 
transparência dessas oportunidades, pois a estatística $t$ mostrou-se muito significativa [ $t$ $(285)=4,378, p<0,001$ ], o que, por conseguinte, leva a ratificação da hipótese 4a.

O parâmetro estimado $b$ da equidade do sistema de remuneração (Equidade_SR) mostrado na tabela dos coeficientes é 0,147 . Esse valor indica que quando a equidade desse sistema aumenta em uma unidade, a motivação intrínseca aumenta 0,147 níveis. $O$ valor $t$ de5,532 com uma probabilidade associada menor que 0,001, $[t(285)=5,532, p<0,001]$, indica ser improvável que o coeficiente de regressão tenha ocorrido por erro amostral. Desta maneira, pode-se afirmar que a mudança nos níveis de motivação intrínseca realmente é divergente se o escore da equidade do sistema de remuneração aumentar em uma unidade. Por conseguinte, pode-se destacar que os níveis de motivação intrínseca dos docentes da UFT aumentam 0,147 graus como resultado do aumento, em média, de uma unidade na equidade do seu sistema de remuneração, corroborando a hipótese 3b. Essa interpretação somente é verdadeira se os efeitos dos outros previsores significativos forem mantidos constantes.

Em relação ao efeito da equidade das oportunidades de progressão (Equidade_OP) sobre a motivação intrínseca, pode-se afirmar com base nos resultados apresentados que os níveis de motivação intrínseca dos docentes da UFT pesquisados não aumentam em decorrência do aumento de uma unidade na equidade dessas oportunidades. O valor da estatística $t$ confirma esses resultados ao mostrar-se de forma não significativa [ $t(272)=$ $0,537, p>0,05]$, levando, dessa maneira, a refutação da hipótese $4 b$.

No que diz respeito à influência da controlabilidade do sistema de remuneração (Controlabilidade_SR) sobre a motivação intrínseca, os resultados permitem dizer que os níveis de motivação intrínseca dos docentes da UFT não aumentam em decorrência do aumento de uma unidade na controlabilidade desse sistema. Como o valor do teste $t$ mostrou não ser significativo, [ $t(272)=1,133, p>0,05]$, a mudança nos níveis de motivação intrínseca dos docentes da UFT não pode ser confirmada, o que, conduz a rejeição da hipótese 3c. No tocante à relação entre a controlabilidade das oportunidades de progressão (Controlabilidade_OP) e a motivação, os resultados encontrados evidenciaram que os níveis de motivação intrínseca dos docentes da UFT também não aumentam em decorrência do aumento de uma unidade na controlabilidade dessas oportunidades, pois o valor da estatística $t$ não é significativo [ $t(272)=0,891, p>0,05]$, levando a rejeição da hipótese $4 c$.

Em relação ao efeito da idade dos docentes sobre a motivação, os resultados mostram que os níveis de motivação intrínseca dos docentes da UFT aumentam 0,009 graus em decorrência do aumento, em média, de uma unidade (no caso um ano) na idade dos docentes. Sobre o efeito que o estado civil do docente exerce sobre a motivação, os resultados indicam que os níveis de motivação intrínseca dos docentes da UFT diminuem

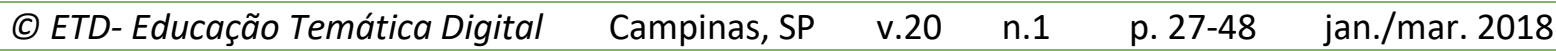


0,072 graus como resultado da mudança da sua condição civil de casado(a) para solteiro(a), de solteiro(a) para viúvo(a), de viúvo(a) para divorciado(a) e de divorciado(a) para união estável.

Em relação à multicolinearidade nos dados, analisando os valores apresentados pelo FIV e pela estatística Tolerância e em paralelo com as observações de Meyers (1990), Menard (2002) e Field (2009), pode-se notar que todos os valores de FIV estão abaixo de 10 e todas as Tolerâncias acima de 0,20 , o que leva a concluir com uma margem considerável de segurança que esses dados não apresentam problemas de colinearidade (altas correlações entre si).

De forma resumida, pode-se destacar que as variáveis transparência das oportunidades de progressão, equidade do sistema de remuneração, idade e estado civil exercem um efeito significativo na motivação intrínseca dos docentes da UFT pesquisados. Porém, as variáveis transparência do sistema de remuneração, equidade das oportunidades de progressão, controlabilidade do sistema de remuneração e controlabilidade das oportunidades de progressão não afetam de forma significativa a motivação intrínseca desses professores.

\section{CONSIDERAÇÕES FINAIS}

Diante da importância que o tema assume para qualquer organização e da escassez de estudos na literatura brasileira, esta pesquisa teve como objetivo avaliar de que forma um sistema de compensação (remuneração e promoção) exerce influência na motivação dos colaboradores docentes da Universidade Federal do Tocantins (UFT).

Os resultados permitiram apontar que $67,3 \%$ da variação nos níveis de motivação extrínseca dos docentes da UFT pode ser atribuída às variáveis transparência das oportunidades de progressão, equidade do sistema de remuneração, equidade das oportunidades de progressão, controlabilidade do sistema de remuneração, tempo de atuação na instituição e existência de metas claras a cumprir na instituição. Essas variáveis evidenciaram uma relação estatisticamente significativa positiva com a motivação extrínseca (à medida que elas aumentam a motivação extrínseca também aumenta), exceto a variável tempo na instituição, que mostrou uma relação negativa, indicando que à medida que vai aumentando o tempo de atuação dos docentes na instituição, sua motivação extrínseca tende a diminuir. Por outro lado, as variáveis independentes transparência do sistema de remuneração e controlabilidade das oportunidades de progressão não afetaram de forma significativa a motivação extrínseca desses professores. 
A influência das características do sistema de compensação na motivação intrínseca do pessoal docente da UFT assegura que $22,3 \%$ da variação nos seus níveis de motivação intrínseca podem ser atribuídas às variáveis transparência das oportunidades de progressão, equidade do sistema de remuneração, idade e estado civil. Essas variáveis exercem um efeito estatisticamente significativo positivo na motivação intrínseca, exceto a variável estado civil, que apresentou uma relação inversa com a variável dependente, indicando que à proporção que a condição civil dos docentes passa de casado(a) para solteiro(a), de solteiro(a) para viúvo(a), de viúvo(a) para divorciado(a) e de divorciado(a) para união estável suas motivações intrínsecas propendem a diminuir. Porém, as variáveis transparência do sistema de remuneração, equidade das oportunidades de progressão, controlabilidade do sistema de remuneração e controlabilidade das oportunidades de progressão não afetam de forma significativa estatisticamente a motivação intrínseca desses professores.

No comparativo entre as variáveis independentes que exercem um efeito significativo sobre a motivação, pode-se apontar que a transparência das oportunidades de progressão e a equidade do sistema de remuneração são variáveis que afetam positivamente tanto a motivação extrínseca quanto a intrínseca dos docentes da UFT. Isso significa que para os docentes da instituição a existência de critérios ou regras claras para se fazer progressão e a justiça na maneira com que eles são remunerados os fazem sentirem energizados, motivados extrínseca e intrinsecamente, bem como lhes proporcionam um sentimento de alegria, contentamento e prazer. Entre as variáveis independentes que não afetam a motivação extrínseca e intrínseca, a transparência do sistema de remuneração e a controlabilidade das oportunidades de progressão são as variáveis que sobressaíram. Isso significa que estas variáveis não influenciam a motivação dos docentes porque provavelmente estão fora do seu alcance, haja vista que a forma como a remuneração é definida e o controle das oportunidades de progressão serem definidos com base em acordos coletivos e leis próprias.

Como limitações desta pesquisa, ressalta-se que os resultados apresentados, diante do universo que poderia ser pesquisado (no caso, professores de todos os sete campi da UFT), limitam-se à amostra utilizada, devendo as generalizações a respeito dos resultados serem realizadas com certa e a devida precaução. Por mais que os modelos de regressão múltiplas utilizados aderiram bem aos dados e satisfizeram algumas condições como normalidade e linearidade nos dados, homogeneidade de variâncias, independência de erros e ausências de multicolinearidade e singularidade (variáveis altamente correlacionadas ou perfeitamente correlacionadas, respectivamente), tirar conclusões além da amostra pode ser um equívoco. 
As conclusões e as limitações desta tese geram várias sugestões para futuras investigações. Assim, este trabalho poderia fornecer um "plano de fundo" para a realização de estudos desta natureza em todos os sete campi da UFT e também em outras universidades tanto públicas quanto particulares, fazendo-se as devidas adaptações e contando-se com a possibilidade de participação de um maior número de respondentes, com o objetivo de poder generalizar os resultados do modelo para além da amostra. Como a análise utilizada integra diversas dimensões, sua perspectiva multidimensional pode contribuir para que novos insights sejam obtidos por meio de outras pesquisas desse tipo.

Como as variáveis independentes não explicaram cem por cento (100\%) da variação nas variáveis dependentes, deve haver outras variáveis que também exercem influência nas variáveis dependentes. Assim, não se pode deixar de mencionar a necessidade de estudos futuros buscarem descobrir ou identificar outros fatores que podem explicar o restante dessa variação nas variáveis dependentes.

Por fim, questões relacionadas à causalidade da relação entre as características do sistema de compensação e a motivação tanto extrínseca quanto intrínseca poderiam ser melhor discutidas, pois existem sérias dúvidas na literatura a respeito de quais os mecanismos operantes na relação entre esses elementos ou variáveis.

\section{REFERÊNCIAS}

ALBUQUERQUE, Lindolfo Galvão de. Estratégia de recursos humanos e competitividade. In: VIEIRA, Marcelo Milano Falcão; OLIVEIRA, Lúcia Maria Barbosa de (Org.). Administração contemporânea: perspectivas e estratégias. São Paulo: Atlas, 1999.

ALVES, Thiago; PINTO, José Marcelino de Rezende. Remuneração e características do trabalho docente no Brasil: um aporte. Cadernos de Pesquisa, São Paulo, v.41, n. 143, p. 606-639, maio/ago., 2011. Disponível em:

http://www.scielo.br/pdf/cp/v41n143/a14v41 n143.pdf. Acesso em: 03 jan. 2016.

BÉNABOU, Roland; TIROLE, Jean. Intrinsic and extrinsic motivation. Review of Economic Studies, v. 70, n. 3, p. 489-520, 2003.Disponível em:

https://www.princeton.edu/ rbenabou /papers/RES2003.pdf. Acesso em: 04 jan. 2016.

BERGAMINI, Cecília Whitaker. Avaliação de desempenho humano na empresa. 4. ed. São Paulo: Atlas, 1992.

BERGAMINI, Cecília Whitaker. Motivação nas organizações. 5. ed. São Paulo: Atlas, 2008.

BOHLANDER, George; SNELL, Scott.Administração de recursos humanos.14 ed. São Paulo: Cengage Learning, 2009.

$\begin{array}{llllll}\text { (C) ETD-Educação Temática Digital } & \text { Campinas, SP } & \text { v.20 } & \text { n.1 } & \text { p. 27-48 } & \text { jan./mar. } 2018\end{array}$


BRYMAN, Alan. Quantity and quality in social research. New York: Routledge, 2004.

CÂMARA, Pedro B. Os sistemas de recompensas e a gestão estratégica de recursos humanos. 3. ed. Porto: Dom Quixote, 2011.

CARDOSO, Maurício Farias; CARDOSO, Janice de Freitas; SANTOS, Simone Rodrigues dos. O impacto da rotatividade e do absenteísmo de pessoal sobre o custo do produto: um estudo em uma indústria gaúcha. Revista de Gestão, Finanças e Contabilidade, Salvador, v. 3, n. 1, p. 107-121, jan./abr., 2013. Disponível em:

http://www.revistas.uneb.br/index. php/financ/article/view/210. Acesso em: 13 fev. 2016.

CASTRO, Alfredo Pires de. Liderança motivacional: como desenvolver pessoas e organizações, através do coaching e da motivação. Rio de Janeiro: Qualitymark, 2008.

CAVALCANTI, Vera Lucia et al.. Liderança e motivação. Rio de Janeiro: FGV, 2005.

CERVO, Amado Luiz; BERVIAN, Pedro Alcino. Metodologia científica. 4. ed. São Paulo: Makron Books, 2002.

CHIANG, Flora T.; BIRTCH, Thomas A. An empirical examination of reward preferences within and across national settings. Management International Review, v. 46, n. 5, p. 573596, 2006.Disponível em: http://link.springer.com/article/10.1007/s11575-006-0116-4. Acesso em: 14 fev. 2016.

DAVIS, Keith; NEWSTROM, John. Comportamento humano no trabalho. São Paulo: Pioneira, 1998.

$\mathrm{DECl}$, Edward L.The effects of contingent and non contingent rewards and controls on intrinsic motivation. Organizational Behavior and Human Performance, v. 8, p. 217-229, 1972.Disponível em: http://selfdeterminationtheory.org/SDT/documents/1972 Deci OBHP.pdf. Acesso em: 16 fev. 2016.

DECl, Edward L.; RYAN, Richard M.. The "what" and "why" of goal pursuits: human needs and self-determination of behavior. Psychological Inquiry, v 11, n 4, p. 227-268, 2000. Disponível em: http://users.ugent.be/ wbeyers/scripties2011/artikels/Deci\&Ryan 2000. pdf. Acesso em: 06 mar. 2016.

DÖRNYEI, Zoltán. Motivation in action: towards a process-oriented conceptualisation of student motivation. British Journal of Educational Psychology, v. 70, p. 519-538, 2000.Disponível em: http://www.zoltandornyei.co.uk/uploads/2000-dornyei-bjep.pdf. Acesso em: 09 mar. 2016.

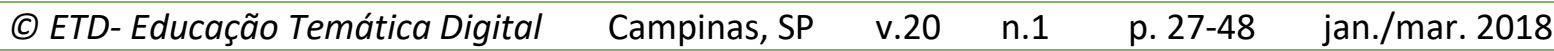


DUARTE, Adriana; MELO, Savana Diniz Gomes.Qualidade da educação e política de remuneração docente: quais as implicações dessa relação? Revista Educação em Questão, Natal, v. 46, n. 32, p. 202-225, mai./ago., 2013. Disponível em:

https://periodicos.ufrn.br/ educacaoemquestao/article/download/5128/4106. Acesso em: 15 abr. 2016.

DURBIN, James R.; WATSON, Geoffrey S. Testing for serial correlation in least squares regression II. Biometrika, v. 38, n. 1-2, p. 159-178, 1951. Disponível em: http://www. saramitchell.org/tsafall2011.pdf. Acesso em: 07 mar. 2016.

DUTRA, Joel Souza. Gestão de pessoas: modelo, processos, tendências e perspectivas. São Paulo: Atlas, 2002.

FERREIRA, Aurélio Buarque de Holanda. Miniaurélio século XXI: o minidicionário da língua portuguesa. 5. ed. Revista e ampliada. Rio de Janeiro: Nova Fronteira, 2001.

FIELD, Andy. Descobrindo a estatística usando o SPSS. Tradução: Lorí Viali. 2. ed. Porto Alegre: Artmed, 2009.

FRANÇA, Ana Cristina Limongi. Práticas de recursos humanos: conceitos, ferramentas e procedimentos. 1. ed. 3. reimpr. São Paulo: Atlas, 2009.

FREY, Bruno S.; JEGEN, Reto. Motivation crowding theory: a survey of empirical evidence. Journal of Economic Surveys, v. 15, n 5, p. 589-611, 2001.Disponível em: ftp://ster. kuleuven.ac.be/incoming/eva/iewwp049.pdf Acesso em: 12 abr. 2016.

GIL, Antônio Carlos. Como elaborar projetos de pesquisa. 4. ed. São Paulo: Atlas, 2002.

GOMES, Dione Fagundes Nunes; AMORIM, Maria Cristina Sanches. Liderança e programas de recompensas: limites e possibilidades. Revista de Negócios, Blumenau, v. 13, n. 3, p. 2636, jul./set., 2008. Disponível em:

http://proxy.furb.br/ojs/index.php/rn/article/viewFile/771/868 Acesso em: 18 mai. 2016

HARRIS, Michael. Human resource management. San Diego: The Dryden, 1997.

HELLRIEGEL, Don; SLOCUM JR., John W. Organizational behavior. 10 ed. Mason OH: SouthWestern, 2004.

JESSUP, Gilbert e Helen. Seleção e avaliação no trabalho. Rio de Janeiro: Zahar, 1977.

KAST, Fremont E.; ROSENZWEIG, James E. Organization and management: a systems approach. Tóquio: McGraw-Hill Kogakusha, 1970.

(C) ETD- Educação Temática Digital Campinas, SP $\quad$ v.20 $\quad$ n.1 $\quad$ p. 27-48 jan./mar. 2018


KIDDER, Louise H. et al. Métodos de pesquisa nas relações sociais. 2. ed. São Paulo: EPU, 1987.

KÖCHE, José Carlos. Fundamentos de metodologia científica: teoria da ciência e iniciação à pesquisa. 20. ed. Petrópolis, RJ: Vozes, 2002.

LAKATOS, Eva Maria; MARCONI, Marina de Andrade. Fundamentos de metodologia científica. 6. ed. São Paulo: Atlas, 2005.

LEITE, Francisco Tarciso. Metodologia científica: iniciação à pesquisa científica, métodos e técnicas de pesquisa, metodologia da pesquisa e do trabalho científico (monografias, dissertações, teses e livros). Fortaleza: Universidade de Fortaleza, 2004.

MALHOTRA, Naresh K. Pesquisa de marketing: uma orientação aplicada. 6 ed. Porto Alegre: Bookman, 2012.

MARRAS, Jean Pierre. Administração de recursos humanos: do operacional ao estratégico. 14. ed. São Paulo: Saraiva, 2011.

MARRAS, Jean Pierre. Administração de recursos humanos: do operacional ao estratégico. 3. ed. São Paulo: Futura, 2000.

MEDEIROS, Rochele Kaline Reis de; ALVES, Rafaela da Cunha; RIBEIRO, Sidney Roberto S. Turnover: uma análise dos fatores que contribuem para a decisão de sair da empresa dos colaboradores da Alfa Comércio Ltda. Revista Científica da Escola de Gestão e Negócios (Connexio), v. 2, n. 1, p. 115-126, 2012. Disponível em: https://repositorio.unp. br/index.php/connexio/article/view/222. Acesso em: 23 mai. 2016.

MENARD, Scott W..Applied logistic regression analysis. Sage universisty paper series on quantitative applications in the social sciences. Thousand Oaks (CA): Sage, p. 7-106, 2002.

MEYERS, Raymond H. Classical and modern regression with applications. Boston (MA): Duxbury Press, 1990.

MILES, Raymond E. Theories of management: implications of organizational behavior and development. Tokyo: McGraw-Hill, 1975.

MITCHELL, Terence R. Motivation: new direction for theory, research and practice. Academy of Management Review, v. 7, n. 1, p. 80-88, 1982. Disponível em: http://www. jstor.org/stable/257251?origin=crossref\&seq=1\#page scan tab contents. Acesso em: 12 abr. 2016.

PETRI, Herbert L. Motivation: theory, research and applications. Pacific Grove, CA: Brooks/Cole Publishing Company, 1996.

(C) ETD- Educação Temática Digital $\quad$ Campinas, SP $\quad$ v.20 $\quad$ n.1 $\quad$ p. 27-48 jan./mar. 2018


RICHARDSON, Roberto Jarryet al. Pesquisa social: métodos e técnicas. 3.ed. São Paulo: Atlas, 1999.

SALGADO, Léo. Motivação no trabalho. Rio de Janeiro: Qualitymark, 2005. STONER, James; FREEMAN, Edward. Administração. 5. ed. Rio de Janeiro: Prentice Hall do Brasil, 1985.

TAMAYO, Alvaro; PASCHOAL, Tatiane. A relação da motivação para o trabalho com as metas do trabalhador. Revista de Administração Contemporânea (RAC),v. 7, n. 4, p. 33-54, out./dez., 2003. Disponível em: http://www.scielo.br/pdf/rac/v7n4/v7n4a03.pdf. Acesso em: 13 abr. 2016.

VAN HERPEN, Martinus Franciscus Marcellis. Pay, promotions, and performance: essays on personnel economics. 2007, 187 f. Tese (Doutorado em Economia). Universidade de Groningen, 2007.

VIEIRA, Lívia Fraga; OLIVEIRA, Tiago Grama. As condições do trabalho docente na educação infantil no Brasil: alguns resultados de pesquisa (2002-2012). Revista Educação em Questão, Natal, v. 46, n. 32, p. 131-154, maio/ago., 2013. Disponível em:

https://periodicos.ufrn.br/educacaoemquestao/article/download/5125/4103. Acesso em: 20 jul. 2016.

VIEIRA, Lívia Maria Fraga; SOUZA, Gizele de. Trabalho e emprego na educação infantil no Brasil: segmentações e desigualdades. Educar em Revista (Número Especial), Curitiba, n. 1, p. 119-139, 2010. Disponível em:

http://www.gestrado.net.br/images/publicacoes/14/Artigo5.pdf. Acesso em: 21 jul. 2016.

WOOD JR., Thomas; PICARELLI FILHO, Vicente. Remuneração estratégica: a nova vantagem competitiva. São Paulo: Atlas, 1999.

\footnotetext{
' Revisão gramatical do texto sob a responsabilidade dos autores.
} 